\title{
A NOTE ON RIGHT INVARIANT INTEGRALS ON LOCALLY COMPACT SEMIGROUPS
}

\author{
U. B. TEWARI
}

(Received 15 December 1966, revised 8 June 1967)

An integral on a locally compact Hausdorff semigroup $S$ is a nontrivial, positive linear functional $\mu$ on the space $K(S)$ of real-valued continuous functions on $S$ with compact support. If $S$ has the property:

$$
A s^{-1}=\{x \mid x \in S \text { and } x s \in A\}
$$

is compact whenever $A$ is compact subset of $S$ and $s \in S$, then the function $f_{a}$ defined by $f_{a}(x)=f(x a)$ is in $K(S)$ whenever $f \in K(S)$ and $a \in S$. An integral on a locally compact semigroup $S$ with the property $(P)$ is said to be right invariant if $\mu\left(f_{a}\right)=\mu(f)$ for all $f \in K(S)$ and $a \in S$.

In [1] Michael has shown that if $S$ is a separable, metric locally compact semigroup with the properties:

(A) for each pair of compact sets $C, D$ of $S$, the set

is compact;

$$
\begin{aligned}
C D^{-1} & =\{x \mid x \in S \text { and } x y \in C \text { for some } y \in D\} \\
& =\bigcup_{y \in D} C y^{-1}
\end{aligned}
$$

(B) for each pair of compact sets $C, D$ of $S$, the set

is compact;

$$
\begin{aligned}
D^{-1} C & =\{x \mid x \in S \text { and } y x \in C \text { for some } y \in D\} \\
& =\bigcup_{y \in D} y^{-1} C
\end{aligned}
$$

(C) $S$ contains exactly one minimal left ideal;

then $S$ admits a right invariant integral.

In this note our aim is to prove the following:

THEOREM 1. If there exists a right invariant integral $\mu$ on a locally compact semigroup $S$ with the properties $(P)$ and $(B)$ then $\int d \mu$ is always infinite unless $S$ is compact in which case $\int d \mu$ is always finite.

Proof. Suppose $S$ is not compact and $\int d \mu<\infty$. We can select $f \in K(S)$ 
such that $\int f d \mu>0$ and $f \geqq 0$. Without loss of generality we can assume that $f \leqq 1$. It suffices to determine a sequence $\left\{s_{n}\right\}$ in $S$ such that the supports of the functions $f_{s_{n}}$ are disjoint. Clearly then, it will follow that $\sum_{i=1}^{n} f_{s_{i}} \leqq 1$ whence $n \int f d \mu \leqq \int d \mu<\infty$ for each $n$. This leads to a contradiction. Let us denote the support of $f$ by $A$. Then the supports of $t_{s_{n}}$ will be contained in $A s_{n}^{-1}$. Choose $s_{1} \in S$ and assume that $s_{1}, s_{2}, \cdots, s_{n}$ have been constructed. Then in order that $A s_{n+1}^{-1}$ be disjoint from $A s_{i}^{-1}(i=1,2, \cdots, n)$, we must have $s_{n+1}$ not belonging to $\bigcup_{i=1}^{n}\left(A s_{i}^{-1}\right)^{-1} A$. Since $S$ is not compact, such an $s_{n+1}$ exists. This completes the proof.

For the next theorem, we need the following result of Rosen [3].

THEOREM 2. There exists a right invariant integral on a compact semigroup $S$ if and only if $S$ contains exactly one minimal left ideal.

Theorem 1 in conjunction with Theorem 2 yields

THEOREM 3. In order that on a locally compact semigroup $S$ with the properties $(P)$ and $(B)$ there exist a right invariant integral $\mu$ with $\int d \mu<\infty$, it is necessary and sufficient that $S$ be compact and contain exactly one minimal left ideal.

Remark. We observe that conditions $(P)$ and $(B)$ of Theorem 1 cannot entirely be dropped. This we show by quoting an example, due to E. Granirer $([5]$, page 58), of a locally compact semigroup in which conditions $(P)$ and $(B)$ do not hold but there exists a right invariant integral with respect to which the semigroup has finite measure.

Let $Z_{n}=\left\{e_{1}, e_{2}, \cdots, e_{n}\right\}$ with the relations $e_{i} \cdot e_{j}=e_{i}$, and let $A$ be a finite group. Then $A \times Z_{n}=\left\{\left(a, e_{i}\right) \mid a \in A\right.$ and $\left.e_{i} \in Z_{n}\right\}$ becomes a semigroup under the multiplication $\left(a, e_{i}\right) \cdot\left(b, e_{j}\right)=\left(a b, e_{i}\right)$. One easily sees that the set $A_{i}=\left\{\left(a, e_{i}\right) \mid a \in A\right\}$ is a finite group isomorphic to $A$. Let $G_{0}$ be an infinite group. In $G=G_{0} \cup\left(A \times Z_{n}\right)$ we define a multiplication $*$ which renders $G$ a semigroup structure as follows: if $g^{\prime}, g^{\prime \prime}$ are both in $G_{0}$ (or both in $A \times Z_{n}$ ) then $g^{\prime} * g^{\prime \prime}$ means multiplication in $G_{0}$ (or in $A \times Z_{n}$ ). If $g^{\prime} \in G_{0}$ and $g^{\prime \prime} \in A \times Z_{n}$, define $g^{\prime} * g^{\prime \prime}=g^{\prime \prime} * g^{\prime}=g^{\prime \prime}$. It is shown in [5] that $A_{1}, A_{2}, \cdots, A_{n}$ are the only subsets of $G$, which simultaneously are finite groups, are right ideals and satisfy the right cancellation law.

Now $G$ has exactly $n$ finite disjoint groups which are right ideals with right cancellation. As in Granirer [4, lemma 3.1], one can prove that $I=\bigcup_{i=1}^{n} A_{i}$ is a finite left ideal in $G . I$ is also a right ideal. Thus $G$ contains a finite ideal $I$.

$G$ endowed with the discrete topology becomes a locally compact semigroup and $I$ a compact ideal in $G$. For $s \in I$,

$$
I s^{-1}=\{x \in G \mid x s \in I\}=G \text { and } s^{-1} I=\{x \in G \mid s x \in I\}=G .
$$


Thus $(P)$ and $(B)$ do not hold in $G$. Define $\mu_{i}(f)=\sum_{a \in A_{i}} f(a)$ for $f \in K(G)$. One can easily see that $\mu_{i}$ is a right invariant integral on $G$ for each $i$. Also the measure of $G$ with respect to $\mu_{i}$ is $N$ for each $i$, where $N$ is the number of elements of $A$. Thus we have shown that $G$ admits a right invariant integral with respect to which $G$ has finite measure.

Incidentally, this gives an example of a locally compact semigroup admitting at least ' $n$ ' different invariant integrals.

The author expresses his thanks to Professor T. P. Srinivasan for his valuable suggestions in preparing this note.

\section{References}

[1] J. H. Michael, 'Right invariant integrals on locally compact semigroup', J. Aust. Math. Soc. 4 (1964), 273-286.

[2] L. Nachbin, The Haar Integral (Van Nostrand Company, New York, 1965).

[3] W. G. Rosen, 'On the invariant means over compact semigroups', Proc. Amer. Math. Soc. 7 (1956), 1076-1082.

[4] E. Granirer, 'On amenable semigroups with a finite dimensional set of invariant means I', Illinois J. of Math. 7 (1963), 32-48.

[5] E. Granirer, 'On amenable semigroups with a finite dimensional set of invariant means II', Illinois J. of Math. 7 (1963) 49-58.

Department of Mathematics

Panjab University

Chandigarh, India 